\title{
Tiếp cận đa chiều về quản lí hoạt động đào tạo ở Đại học Quốc gia Hà Nội
}

\author{
Vương Thị Phương Thảo ${ }^{1, *}$, Phan Xuân Hiếu ${ }^{2}$ \\ ${ }^{I}$ Trung tâm Khảo thí Đại học Quốc gia Hà Nội \\ ${ }^{2}$ Truò̀ng Đại học Công nghệ, Đại học Quốc gia Hà Nội \\ Nhận ngày 26 tháng 9 năm 2016 \\ Chỉnh sửa ngày 16 tháng 02 năm 2017; Chấp nhận đăng ngày 15 tháng 03 năm 2017
}

\begin{abstract}
Tóm tắt: Theo tiếp cận đa chiều về đảm bảo chất lượng giáo dục, bài báo này khảo sát, đánh giá công tác quản lí đào tạo đại học ở Đại học Quốc gia Hà Nội một cách toàn diện (đa tham số) và khách quan (đa phương pháp, đa nguồn). Trong đó, đánh giá của cán bộ được thực hiện qua 8 nhân tố với 48 tiêu chí bao gồm: mục tiêu và kế hoạch đào tạo; quản lí $(\mathrm{QL})$ chương trình đào tạo; tuyển sinh; tổ chức thực hiện đào tạo; QL hoạt động giảng dạy của giảng viên; QL hoạt động học tập của sinh viên; QL đội ngũ cán bộ quản lí, nhân viên hỗ trợ hoạt động đào tạo; QL môi trường học tập, cơ sở vật chất và trang thiết bị. Đánh giá của sinh viên được thể hiện qua 4 nhân tố: nội dung chương trình đào tạo; hoạt động giảng dạy; hoạt động đào tạo; môi trường, cơ sở vật chất, thiết bị phục vụ giảng dạy và học tập. Các kết quả nghiên cứu đã được củng cố, khẳng định qua so sánh với kết quả đánh giá kiểm định điều kiện đảm bảo chất lượng của các chuyên gia mạng lưới các đại học ASEAN. Tám khuyến nghị được đưa ra để tăng cường điều kiện đảm bảo chất lượng đào tạo.
\end{abstract}

Tù khóa: Tiếp cận đa chiều, quản lí đào tạo, kiểm định chất lượng giáo dục, Đại học Quốc gia Hà Nội.

\section{1. Đặt vấn đề}

Chất lượng đào tạo là mối quan tâm và chỉ số quan trọng của cơ sở giáo dục đại học đối với tất cả các bên liên quan - từ giảng viên và người học đến các nhà quản lí và tuyển dụng. Chất lượng đào tạo không phải là một chỉ số có tính học thuật một chiều (one-dimentional notion). Theo tiếp cận về yêu cầu và mong muốn của các bên liên quan, chất lượng đào tạo là một khái niệm đa chiều (multi-dimentional concept) $[1,2]$. Do đó, việc đánh giá chất lượng đào tạo cũng cần được triển khai một cách toàn diện (đa tham số) và khách quan (đa phương pháp, đa nguồn).

\footnotetext{
Tác giả liên hệ. ĐT.: 84-912103589.

Email: thaovtp@vnu.edu.vn
}

Trong tuyên bố thế giới về Tầm nhìn và hành động của giáo dục đại học thế kỉ 21 (The World Declaration on Higher Education for the Twenty First Century: Vision and Action) [2], nội dung đánh giá chất lượng đào tạo đại học bao gồm tất cả chức năng và hoạt động đào tạo: từ chương trình đào tạo, tổ chức đào tạo và nghiên cứu, cán bộ, người học đến lớp học và môi trường học thuật, đặc biệt có cả nội dung về phục vụ cộng đồng. Đánh giá chất lượng đào tạo được thực hiện thông qua đánh giá trong (tự đánh giá) và đánh giá ngoài (chuyên gia từ bên ngoài) một cách đọc lập. Bên cạnh đó, còn có các nghiên cứu độc lập của các nhà khoa học.

Trong những năm gần đây, việc đánh giá trong và đánh giá ngoài đã bắt đầu được thực hiện trong khuôn khổ các hoạt động kiểm định điều kiện đảm bảo chất lượng giáo dục ở cấp 
chương trình [1] và cấp cơ sở giáo dục đại học [3]. Các hoạt động này có nhiều ưu điểm và lợi thế như: bộ tiêu chí được chuẩn hóa và áp dụng chung cho toàn quốc gia (Việt Nam), khu vực (ASEAN) hoặc hiệp hội (ABET)...; được triển khai một cách chính ngạch, có tính chuyên nghiệp cao. Tuy nhiên, do mục tiêu áp dụng chung cho một số đông, các phương pháp này cũng bộc lộ một số hạn chế như không phản ánh được các tiêu chí đặc thù của các cơ sở giáo dục, mục tiêu đánh giá riêng của nhà quản lí và nghiên cứu; chỉ áp dụng phương pháp nghiên cứu tài liệu, khảo sát thực địa và phỏng vấn đại diện nên số lượng đối tượng khảo sát ít; không được bảo mật nên tính khách quan có thể hạn chế.

Nghiên cứu này tiếp cận khảo sát chất lượng quản lí đào tạo (QLĐT) theo phương pháp điều tra thông qua phiếu hỏi và áp dụng ở Đại học Quốc gia Hà Nội (ĐHQGHN) - một mô hình đại học hai cấp, có tính tự chủ và đặc thù cao; có tính độc lập tương đối với hệ thống giáo dục của cả nước. Các kết quả nhận được trong nghiên cứu này được phân tích và so sánh với một số nguồn khảo sát khác, là cơ sở để tác giả đề xuất một số kiến nghị phục vụ cho việc tăng cường công tác quản lí nâng cao chất lượng đào tạo đại học.

\section{Phương pháp nghiên cứu và mẫu nghiên cứu}

\subsection{Phuơng pháp nghiên cứu}

Phương pháp nghiên cứu như nghiên cứu lý luận (phân tích các tài liệu, văn bản quản lí điều hành liên quan đến QLĐT đại học) phối hợp với nghiên cứu thực tiễn (điều tra các đối tượng là cán bộ $\mathrm{QL}$, giảng viên, sinh viên) $[4,5]$.

Trong nghiên cứu này, 8 nội dung (nhân tố) sau đây được quan tâm khảo sát: (i) Mục tiêu và kế hoạch đào tạo; (ii) QL chương trình đào tạo; (iii) Tuyển sinh; (iv) Tồ chức thực hiện đào tạo; (v) QL hoạt động giảng dạy của giảng viên; (vi) QL hoạt động học tập của sinh viên; (vii) QL đội ngũ cán bộ quản lí, nhân viên hỗ trợ hoạt động đào tạo; và (viii) $\mathrm{QL}$ môi trường học tập, cơ sở vật chất và trang thiết bị.
Căn cứ vào kết quả của quá trình xây dựng thang đo, các tiêu chí đã được cụ thể hóa thành các chỉ báo, làm cơ sở để đề xuất, dự thảo mẫu phiếu điều tra. Mẫu phiếu dự thảo này được một số chuyên gia đóng góp ý kiến; sau đó được bổ sung, điều chỉnh trước khi tiến hành khảo sát thử nghiệm. 2 mẫu phiếu hỏi theo thang đo Likert 5 mức độ đã được hoàn chỉnh. Tổng số 136 phiếu đã dành cho cán bộ và 482 phiếu dành cho sinh viên thuộc các đơn vị đào tạo trong ĐHQGHN, bao gồm 6 trường đại học thành viên và 2 khoa trực thuộc.

Các thang đo được đánh giá thông qua: hệ số tin cậy Cronbach Alpha và phương pháp phân tích nhân tố EFA. Cụ thể:

- Để đo lường mức độ đáp ứng của công tác QLĐT đại học, khái niệm chỉ số công tác QLĐT đại học và chỉ số đáp ứng của công tác QLĐT đại học đã được sử dụng. Đây là các thống kê được tính toán, tổng hợp dựa theo các phương pháp mô hình hóa toán học (phân tích nhân tố, phân tích độ tin cậy của thang đo, phân tích hồi quy, trung bình số học...). Chỉ số đáp ứng của thành tố được tính bằng giá trị trung bình cộng các biến quan sát thuộc từng thành tố.

- Hệ số Cronbach's Alpha đối với từng thang đo đã được tiến hành tính toán để phân tích độ tin cậy của thang đo về hoạt động đào tạo đại học. Kết quả phân tích cho thấy, hầu hết các thang đo đều có hệ số Cronbach's Alpha lớn hơn 0,75 (mức cao). Kết quả Cronbach's Alpha của từng thành tố dao động trong khoảng $[0,756 ; 0,909]$ và các hệ số tương quan biến tổng của các biến đạt từ 0,471 trở lên. Việc loại bỏ bất kì biến quan sát nào trong thang đo đều làm cho hệ số Cronbach's Alpha trở nên kém ý nghĩa hơn (hệ số Cronbach's Alpha từng khái niệm thành phần giảm đi). Kết quả đánh giá độ tin cậy của bảng hỏi cán bộ về thực trạng công tác QLĐT là 0,972 (số lượng câu hỏi là 48).

- Nhân tố khám phá (EFA) theo phương pháp trích Principals axis factoring kết hợp với phương pháp xoay Varimax cũng đã được phân tích [5]. Các nhân tố (khái niệm) sau khi được kiểm tra đánh giá bằng phương pháp EFA gồm 
8 nhân tố đã nêu với 48 biến quan sát. Sau khi phân tích hệ số tin cậy Alpha, 8 thang đo được đánh giá tiếp theo bằng phương pháp phân tích yếu tố EFA. Kết quả EFA cho thấy thang đo đều đạt yêu cầu về nhân tố trích, phương sai trích $(>50 \%)$ và trọng số nhân tố $(>0,50)$, không có biến nào bị loại.

Như vậy, thông qua đánh giá sơ bộ bằng mô hình Rasch (phần mềm QUEST), hệ số Cronbach Alpha và phân tích nhân tố khám phá EFA, 8 thang đo này đều đạt yêu cầu. Các biến quan sát của thang đo này sẽ được sử dụng trong nghiên cứu chính thức. Bộ công cụ đo lường mức độ ảnh hưởng của các yếu tố đến sự hài lòng của sinh viên đối với hoạt động đào tạo đại học đảm bảo độ tin cậy và độ giá trị của thang đo.

\subsection{Mẫu nghiên cứu}

\subsubsection{Mẫu nghiên cứu đối với cán bộ}

Trong tổng số 136 phiếu dành để khảo sát ý kiến cán bộ, tî lệ cán bộ quản lí là nam chiếm $55,9 \%$; cán bộ quản lí là nữ chiếm 43,4\% (bỏ sót $0,7 \%$ ). Đội ngũ cán bộ trẻ (dưới 35) tại đơn vị chiếm tỉ lệ cao nhất 48,5\% (66 cán bộ). Nhóm cán bộ từ 36 đến 45 tuổi có 53 cán bộ chiếm tỉ lệ 39\%. Đối với nhóm độ tuổi từ 46 đến 55 chiếm khoảng $8,1 \%$. Còn đối tượng quản lí trong độ tuổi từ 56 đến 62 tuổi chỉ chiếm tỉ lệ 3,7\%.

Xét về thâm niên công tác, những cán bộ có thâm niên công tác từ 11 đến 20 năm chiếm tỉ lệ cao nhất 36,8\% (50 cán bộ); những cán bộ có thâm niên công tác từ 10 năm trở xuống chiếm khoảng 27,5\%; những cán bộ có thâm niên công tác từ 21 đến 30 năm có 9 cán bộ chiếm tỉ lệ $6,6 \%$; Còn những cán bộ có thâm niên từ 31 đến 40 năm rất ít chiếm tỉ lệ $1,5 \%$.

Về vị trí công tác trong QLĐT: cấp trường chiếm tỉ lệ cao nhất $60,3 \%$ (có 82 cán bộ QL); cấp khoa có 15 cán bộ QL chiếm tỉ lệ $11 \%$, cấp bộ môn có 28 cán bộ QL chiếm tỉ lệ $20,6 \%$. Tuy nhiên, chúng tôi đã bỏ sót 11 cán bộ quản lí chiếm tỉ lệ $8,1 \%$.

\subsubsection{Mẫu nghiên cứu đối với sinh viên}

Thông tin về đặc điểm mẫu nghiên cứu sinh viên được trình bày trong Bảng 1 . Kết quả phân tích đặc điểm mẫu nghiên cứu cho thấy đa số đối tượng người học được hỏi là nữ giới (chiếm $66,0 \%$ ) và có độ tuổi trong khoảng từ 18 đến 25 tuổi. Đặc điểm khí chất của người học thuộc các kiểu khí chất đa dạng và phân bố đồng đều nhau.

Bảng 1. Đặc điểm mẫu nghiên cứu sinh viên

\begin{tabular}{clll}
\hline Đặc điểm mẫu nghiên cứu & Số lượng & Tỉ lệ (\%) \\
\hline \multirow{3}{*}{ Giới tính } & Nam & 162 & 33,6 \\
& Nữ & 318 & 66,0 \\
& Bỏ sót & 2 & 0,4 \\
Tuổi & $18-20$ & 271 & 56,2 \\
sinh học & $21-25$ & 205 & 42,5 \\
& $26-29$ & 1 & 0,2 \\
& Bỏ sót & 5 & 1,0 \\
& Đại học Công nghệ & 57 & 11,8 \\
& Đại học Giáo dục & 26 & 5,4 \\
& Đại học Khoa học Tự nhiên & 94 & 19,5 \\
Trường/ Khoa & Đại học KHXH\&NV & 35 & 7,3 \\
đang theo học & Đại học Kinh tế & 49 & 10,2 \\
& Đại học Ngoại ngữ & 89 & 18,5 \\
& Khoa Luật & 52 & 10,8 \\
& Khoa Quốc tế & 80 & 16,6 \\
& Bỏ sót & 25 & 5,2 \\
\hline
\end{tabular}




\begin{tabular}{clll}
\hline Đặc điểm mẫu nghiên cứu & Số lượng & Tỉ lệ (\%) \\
\hline \multirow{5}{*}{ Tuýp người } & Năng động, sôi nổi & 217 & 20,1 \\
& Trầm tính & 191 & 17,7 \\
& Có khả năng làm việc nhóm & 212 & 19,6 \\
& Thích nghiên cứu & 154 & 14,3 \\
& Thích thực hành & 301 & 27,9 \\
& Khác & 5 & 0,5 \\
\hline
\end{tabular}

\section{Kết quả và phân tích}

\section{1. Đánh giá của cán bộ về thưc trạng công tác $Q L Đ T$}

3.1.1. Mức độ quan tâm của cán bộ đến hoạt động QLĐT

Kết quả thống kê liên quan đến mức độ quan tâm của cán bộ về hoạt động QLĐT được trình bày trong Bảng 2 ; sự quan tâm này có điểm trung bình (ĐTB) khá cao, dao động từ khoảng 4,22 đến 4,53. Bên cạnh đó, độ lệch chuẩn cũng chỉ dao động trong khoảng 0,6 đến 0,8 . Trong đó, hoạt động QLĐT cấp bộ môn có điểm trung bình cao nhất $(4,53)$ cùng với độ lệch chuẩn thấp nhất $(0,60)$. Điều này phản ánh quan niệm và đánh giá về vai trò và đóng góp của bộ môn trong quy trình đào tạo và đảm bảo chất lượng đào tạo. Đồng thời, đó cũng là mức độ quan tâm của cán bộ quản lí cấp bộ môn đội ngũ cán bộ vừa quản lí, vừa tham gia giảng dạy trực tiếp đến hoạt động QLĐT.

Bảng 2. Kết quả thống kê liên quan đến mức độ quan tâm của cán bộ đến hoạt động QLĐT

\begin{tabular}{lcc}
\hline Các vấn đề quan tâm & ĐTB & Độ lệch chuẩn \\
\hline 1. Hoạt động QLĐT cấp & 4,22 & 0,80 \\
ĐHQGHN & 4,45 & 0,68 \\
2. Hoạt động QLĐT cấp Trường & 4,50 & 0,67 \\
3. Hoạt động QLĐT cấp Khoa & 4,53 & 0,60 \\
4. Hoạt động QLĐT cấp Bộ môn & 4,32 & 0,61 \\
5. Khác & & \\
\hline
\end{tabular}

3.1.2. Mức độ quan tâm và tiếp cận của cán bộ đối với các quy định về công tác QLĐT

Theo thông số thống kê liên quan đến mức độ quan tâm và tiếp cận của cán bộ đối với các quy định về công tác QLĐT tại Bảng 3, ĐTB của mức độ tiếp cận đối với các văn bản hướng dẫn liên quan đến Luật Giáo dục và Luật Giáo dục đại học có ĐTB thấp nhất là 4,15 . Mức độ tiếp cận và nắm các nội dung hướng dẫn và kể hoạch, chiến lược phát triển đào tạo của ĐHQGHN, của Trường và Khoa có $\mathrm{ÐTB}$ cao nhất là 4,42 với độ lệch chuẩn tương ứng là 0,60 và 0,63 . Điều này cho thấy các hướng dẫn về QLĐT cũng như kế hoạch, chiến lược về đào tạo của của ĐHQGHN, Trường, Khoa được cán bộ hiểu rõ nhất, phản ánh chính xác và phù hợp với đặc thù QLĐT ở ĐHQGHN. Trong thực tế, ĐHQGHN thực hiện việc QLĐT theo Quy chế đào tạo riêng với một số quy định đặc thù phù hợp với đặc điểm của mô hình đại học hai cấp. Đồng thời, thực hiện sứ mệnh của mình, ĐHQGHN được phép tiên phong thực hiện một số yếu tố quản trị đại học tiến tiến, trao đổi và hội nhập... Do đó, là những người quản lí trực tiếp, cán bộ có sự quan tâm ưu tiên nhất đối với các văn bản quy định, hướng dẫn, kế hoạch và chiến lược phát triển của ĐHQGHN, tiếp đó mới đến các văn bản Luật Giáo dục, Luật Giáo dục đại học hay các văn bản quy định về QLĐT của Bộ GD\&ĐT. 
Bảng 3. Kết quả thống kê liên quan đến mức độ quan tâm và tiếp cận của cán bộ đối với các quy định về công tác QLĐT

\begin{tabular}{lcc}
\hline Hệ thống văn bản liên quan đến công tác QLĐT & ĐTB & Độ lệch chuẩn \\
\hline 1. Các văn bản Luật Giáo dục và Luật Giáo dục đại học & 4,15 & 0,69 \\
2. Các Quy định về QLĐT của Bộ GD\&ĐT & 4,24 & 0,61 \\
3. Các Quy định về QLĐT của ĐHQGHN & 4,39 & 0,62 \\
4. Các kế hoạch, chiền lược về đào tạo của ĐHQGHN, Trường, Khoa & 4,42 & 0,63 \\
5. Các hướng dẫn về QLĐT của ĐHQGHN, Trường, Khoa & 4,42 & 0,60 \\
\hline
\end{tabular}

3.1.3. Đánh giá của cán bộ đối với hoạt động QLĐT của ĐHQGHN

Như đã nêu ở trên, trong nghiên cứu này, công tác quản lí được khảo sát qua 8 nhân tố: Mục tiêu và kế hoạch đào tạo; QL chương trình đào tạo; Tuyển sinh; Tổ chức thực hiện đào tạo; QL hoạt động giảng dạy của giảng viên; QL hoạt động học tập của sinh viên; QL đội ngũ cán bộ quản lí, nhân viên hỗ trợ hoạt động đào tạo; và QL môi trường học tập, cơ sở vật chất và trang thiết bị. Kết quả phân tích tương quan của cán bộ về hoạt động QLĐTcủa từng đơn vị đào tạo trong ĐHQGHN có sự khác biệt với độ tin cậy $99 \%(\mathrm{p}<0,01)$. Điều đó phản ánh tính thống nhất trong đa dạng của các đơn vị đào tạo ở ĐHQGHN. Các đơn vị có đặc thù, nguồn lực và điều kiện khác nhau xác định giải pháp ưu tiên trong hoạt động QLĐT nhằm hướng tới nâng cao chất lượng đào tạo.

Kết quả khảo sát tổng quát việc đánh giá của cán bộ về hoạt động QLĐT đại học tại ĐHQGHN được biểu diễn trên Hình 1. Nhận thấy rằng, nhân tố quản lí $\mathrm{CTĐT} \mathrm{có} \mathrm{điểm} \mathrm{trung}$ bình cao nhất (ĐTB) là 4,11 chiếm $12,8 \%$. Tiếp theo là các tiêu chí về Mục tiêu và kế hoạch đào tạo, Tuyển sinh, Tổ chức thực hiện đào tạo, QL hoạt động giảng dạy của giảng viên, QL hoạt động học tập của sinh viên, đều có ĐTB cao, dao động từ 4,07 đến 4,09 . Hai nhân tố QL đội ngũ cán bộ quản lí, nhân viên hỗ trợ hoạt động đào tạo; và $\mathrm{QL}$ môi trường học tập, cơ sở vật chất và trang thiết bị có ĐTB thấp hơn cả. Các kết quả này được trình bày và thảo luận chi tiết dưới đây, trong đó tập trung vào các điểm bất cập nhất, cần được quan tâm để cải thiện chất lượng đào tạo.

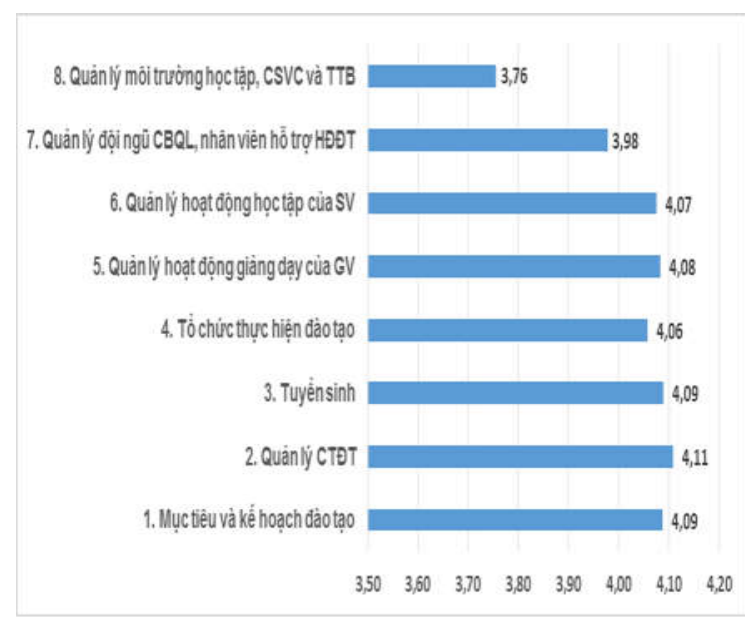

Hình 1. Kết quả khảo sát tổng quát của cán bộ về hoạt động QLĐT đại học tại ĐHQGHN.

Về muc tiêu và kế hoạch đào tạo, tiêu chí đánh giá thứ nhất: Mục tiêu đào tạo được xác định rõ ràng, phù hợp có ĐTB cao $(4,16)$, thuộc tốp 4 các tiêu chí có ĐTB (từ 4,16-4,19) cao nhất trong số 48 tiêu chí khảo sát. Mặc dù cũng có ĐTB cao $(4,04)$, nhưng Tiêu chí có ĐTB thấp nhất trong nhân tố này là tiêu chí 3 : Chiến lược, kế hoạch đào tạo được xây dựng cụ thể, phản ánh mức độ hài lòng chưa cao của cán bộ.

Bảng 4. Thống kê kết quả khảo sát của cán bộ về mục tiêu và kế hoạch đào tạo

\begin{tabular}{lcc}
\hline Mục tiêu và kế hoạch đào tạo & ĐTB & Độ lệch chuẩn \\
\hline 1. Mục tiêu đào tạo được xác định rõ ràng, phù hợp & 4,16 & 0,63 \\
2. Chiến lược, kế hoạch đào tạo được xây dựng cự thể & 4,04 & 0,64 \\
3. Mục tiêu, chiến lược, kế hoạch đào tạo được điều chỉnh phù hợp & 4,07 & 0,65 \\
\hline
\end{tabular}


Về quản lí chưong trình đào tạo, là nhân tố có ĐTB cao nhất, trong đó có hai tiêu chí thuộc tốp 4 các tiêu chí có ĐTB cao nhất (Tiêu chí 6 : Thông tin về CTĐT được công bố rộng rãi và Tiêu chí 9: Yêu cầu về chuẩn đầu ra của CTĐT được xác định rõ ràng, cụ thể). Đây là một kết quả phù hợp với các thống kê khác về đánh giá hệ thống website của ĐHQGHN ngay cả trên các bảng xếp hạng quốc tế như Webometrics [6], 4IUC [7],... Điểm đáng lưu ý ở đây là tiêu chí 7: Nội dung CTĐT được thường xuyên cập nhật và điều chỉnh phù hợp đang có điểm thấp nhất.

Bảng 5. Thống kê kết quả khảo sát của cán bộ về quản lí chương trình đào tạo

\begin{tabular}{lcc}
\hline Quản lý chương trình đào tạo & ĐTB & Độ lệch chuẩn \\
\hline 4. Có văn bản quy định/hướng dẫn xây dựng CTĐT & 4,10 & 0,66 \\
5. CTĐT có mục tiêu rõ ràng, có cấu trúc hợp lí & 4,10 & 0,72 \\
6. Yêu cầu về chuẩn đầu ra của CTĐT được xác định rõ ràng, cụ thể & 4,17 & 0,65 \\
7. Nội dung CTĐT được thường xuyên cập nhật và điều chỉnh phù hợp & 4,03 & 0,71 \\
8. CTĐT được định kì đánh giá bởi các bên liên quan & 4,07 & 0,69 \\
9. Thông tin về CTĐT được công bố rộng rãi & 4,19 & 0,66 \\
\hline
\end{tabular}

Về tuyển sinh, phù hợp với sự quan tâm và đánh giá cao của cộng đồng trong những năm qua về đổi mới tuyển sinh đánh giá theo năng lực, nhân tố tuyển sinh trong khảo sát cũng thuộc nhóm chỉ tiêu có ĐTB cao. Tuy nhiên, Tiêu chí 11: Kế hoạch tuyển sinh được xây dựng cụ thể, phù hợp với thực tế hiện đang có ĐTB thấp $(4,05)$.

Bảng 6 . Thống kê kết quả khảo sát của cán bộ về tuyển sinh

\begin{tabular}{lcc}
\hline Tuyển sinh & ĐTB & Độ lệch chuẩn \\
\hline 10. Nhà trường có thông báo tuyển sinh rõ ràng và rộng rãi & 4,13 & 0,64 \\
11. Kế hoạch tuyển sinh được xây dựng cụ thể, phù hợp với thực tế & 4,05 & 0,68 \\
12. Phương thức tuyển sinh được xây dựng phù hợp với yêu cầu đổi mới & 4,08 & 0,60 \\
13. Tuyển sinh được đối tượng phù hợp với yêu cầu của CTĐT & 4,09 & 0,67 \\
\hline
\end{tabular}

Về tổ chức thực hiện đào tạo, thông số thống kê về Tổ chức thực hiện đào tạo qua đánh giá của cán bộ cho thấy tiêu chí 17: Việc triển khai đào tạo trong ĐHQGHN được thực hiện theo đúng kế hoạch, đúng quy chế có ĐTB $(4,10)$ cao nhất. Bên cạnh đó, tiêu chí 20 và 21 cũng có ĐTB cao, điều đó cho thấy việc kiểm tra, giám sát hoạt động đào tạo được thực hiện nghiêm túc nên công tác QLĐT của ĐHQGHN thật sự có kỉ cương và nề nếp; cùng với việc thực hiện tốt công tác lấy ý kiến phản hồi của các bên liên quan về hoạt động đào tạo nên đã mang lại hiệu quả và chất lượng đào tạo. Tuy nhiên, ĐHQGHN nên quan tâm đến Tiêu chí 15: Chức năng, trách nhiệm và quyền hạn của các bộ phận, cán bộ quản lí, giảng viên và nhân viên được phân định rõ ràng, hiện đang có điểm thấp nhất $(4,01)$.
Về quản lí hoạt động giảng dạy của giảng viên, kết quả đánh giá của cán bộ cho thấy bên cạnh tiêu chí 29: Kiểm tra, giám sát hoạt động giảng dạy của giảng viên được thực hiện nghiêm túc có ĐTB cao nhất là 4,15 , Tiêu chí 26: Giảng viên thực hiện đổi mới phương pháp giảng dạy (vận dụng phương pháp dạy học tích cực, phát huy tính chủ động, sáng tạo của $\mathrm{SV}$; sử dụng các phương tiện kĩ thuật hiện đại để hỗ trợ bài giảng...) có ĐTB thấp nhất là 4,02. Các tiêu chí đánh giá còn lại có ĐTB dao động trong khoảng từ 4,02 đến 4,15 . Nhờ có sự kiểm tra, giám sát hoạt động giảng dạy một cách chặt chẽ nên việc giảng dạy của giảng viên được thực hiện theo đúng nội dung và chương trình đào tạo đã quy định. Nội dung và thời lượng chương trình đào tạo được đảm bảo đầy đủ là 
một trong những yếu tố góp phần đảm bảo chất lượng đào tạo. Điểm yếu nhận được từ khảo sát này có vẻ không phù hợp với xu thế của thời đại đại học 2.0 và nhất là với một đại học được coi là tiên phong đổi mới của Việt Nam (Bảng 8).

Bảng 7. Thống kê kết quả khảo sát của cán bộ về tổ chức đào tạo

\begin{tabular}{|c|c|c|}
\hline Tổ chức thực hiện đào tạo & ĐТВ & $\begin{array}{l}\text { Độ lệch } \\
\text { chuẩn }\end{array}$ \\
\hline 14. Có hệ thống văn bản tố chức, quản lí đào tạo rõ ràng, nhất quán & 4,07 & 0,64 \\
\hline $\begin{array}{l}\text { 15. Chức năng, trách nhiệm và quyền hạn của các bộ phận, cán bộ quản lí, giảng } \\
\text { viên và nhân viên được phân định rõ ràng }\end{array}$ & 4,01 & 0,75 \\
\hline 16. Sự phối hợp giữa các đơn vị trong nhà trường và khoa/bộ môn đạt hiệu quả & 4,04 & 0,66 \\
\hline 17. Việc triển khai đào tạo được thực hiện theo đúng kế hoạch, đúng quy chế & 4,10 & 0,67 \\
\hline 18. Hình thức đào tạo theo tín chỉ đáp ứng yêu cầu học tập của người học & 4,05 & 0,74 \\
\hline $\begin{array}{l}\text { 19. Quy trình và phương pháp và kiểm tra đánh giá quá trình học tập của người } \\
\text { học đảm bảo chính xác, công bằng và khách quan }\end{array}$ & 4,04 & 0,70 \\
\hline $\begin{array}{l}\text { 20. Việc kiểm tra, giám sát hoạt động đào tạo được thực hiện nghiêm túc, mang lại } \\
\text { hiệu quả }\end{array}$ & 4,09 & 0,66 \\
\hline $\begin{array}{l}\text { 21. Việc lấy ý kiến phản hồi của các bên liên quan về hoạt động đào tạo được thực } \\
\text { hiện định kì }\end{array}$ & 4,08 & 0,62 \\
\hline 22. Việc lấy ý kiến phản hồi về người học sau tốt nghiệp được định kì thực hiện & 4,04 & 0,72 \\
\hline
\end{tabular}

Bảng 8 . Thống kê kết quả khảo sát của cán bộ về quản lí hoạt động giảng dạy của giảng viên

\begin{tabular}{lcc}
\hline Quản lí hoạt động giảng dạy của giảng viên & $\begin{array}{c}\text { ĐTB } \\
\text { Độ lệch } \\
\text { chuẩn }\end{array}$ \\
\hline 23. Có đủ đội ngũ giảng viên tham giảng dạy theo quy định đối với mỗi CTĐT & 4,07 & 0,69 \\
24. Đội ngũ giảng viên có năng lực chuyên môn đáp ứng yêu cầu & 4,08 & 0,67 \\
25. Đội ngũ giảng viên được phân công giảng dạy theo chuyên môn được đào tạo & 4,07 & 0,65 \\
$\begin{array}{l}\text { 26. Giảng viên thực hiện đổi mới phương pháp giảng dạy (vận dụng phương pháp dạy học } \\
\text { tích cực, phát huy tính chủ động, sáng tạo của sinh viên; sử dụng các phương tiện kĩ thuật }\end{array}$ & 4,02 & 0,66 \\
hiện đại để hỗ trợ bài giảng...) & 4,07 & 0,60 \\
27. Giảng viên giảng dạy theo đúng nội dung và thời lượng của CTĐT & 4,12 & 0,66 \\
28. Giảng viên thực hiện việc kiểm tra, đánh giá môn học theo đúng đề cương môn học & 0,64 \\
29. Việc kiểm tra, giám sát hoạt động giảng dạy của giảng viên được thực hiện nghiêm túc & 4,15 & 0,15 \\
\hline
\end{tabular}

Về quản lí hoạt động học tập của sinh viên, quản lí kết quả học tập của sinh viên cũng rất quan trọng vì kêt quả học tập phản ánh quá trình học tập của mỗi sinh viên, từ đó đánh giá được chất lượng giảng dạy và đào tạo. Việc quản lí kết quả học tập theo quy định và có thông báo kịp thời cho người học là rất quan trọng và cần thiết, phản ánh tính khách quan, chính xác của việc kiểm tra đánh giá, ĐTB của tiêu chí 36 này đạt cao nhất $(4,17)$ cùng với độ lệch chuẩn thấp nhất $(0,62)$ đã cho thấy hoạt động này được các đơn vị đào tạo thực hiện nghiêm túc nhất.
Về QL đội ngũ cán bộ quản lí, nhân viên hỗ trợ hoạt động đào tạo, theo thống kê liên quan đển QL đội ngũ cán bộ quản lí, nhân viên hỗ trợ hoạt động đào tạo thì độ lệch chuẩn thấp nhất $(0,69)$ của chỉ tiêu Có đủ đội ngũ cố vân học tập, nhân viên để thực hiện các hoạt động hỗ trợ đào tạo so với các chỉ tiêu khác. Kết quả khảo sát cũng cho thấy cần phải có đầy đủ đội ngũ cố vấn học tập và nhân viên để thực hiện các hoạt động hỗ trợ đào tạo. Mặt khác, xét về tiêu chí ĐTB thì chỉ tiêu Đội ngũ cán bộ QL có phẩm chất đạo đức, năng lực quản lí, lãnh đạo có số điểm cao nhất là 4,06 chênh lệch với Đội ngũ 
cố vấn học tập, nhân viên để thực hiện các hoạt động hỗ trợ đào tạo là 0,01 nhưng tiêu chí này nêu rõ điều kiện cần của đội ngũ cán bộ khi thực hiện hoạt động đào tạo là phải phẩm chất đạo đức, năng lực quản lí, lãnh đạo. Tuy nhiên, các vấn đề về chính sách, quyền lợi đối với đội ngũ cán bộ chưa được đánh giá cao và cần được chú trọng hơn nữa (Bảng 10).

Bảng 9. Thống kê kết quả khảo sát của cán bộ về quản lí hoạt động học tập của sinh viên

\begin{tabular}{|c|c|c|}
\hline Quản lí hoạt động học tập của sinh viên & ĐТВ & $\begin{array}{c}\text { Độ lệch } \\
\text { chuẩn }\end{array}$ \\
\hline $\begin{array}{l}\text { 30. Sinh viên được hướng dẫn đầy đủ về CTĐT, các quy định về kiềm tra đánh giá và } \\
\text { các quy định trong quy chế đào tạo, quy chế công tác học sinh, sinh viên }\end{array}$ & 4,01 & 0,63 \\
\hline $\begin{array}{l}\text { 31. Các hoạt động hỗ trợ trong quá trình học tập được Trường/Khoa triển khai thực } \\
\text { hiện và đáp ứng nhu cầu của học tập của sinh viên }\end{array}$ & 4,04 & 0,71 \\
\hline $\begin{array}{l}\text { 32. Trường/Khoa triển khai tốt công tác giáo dục mục đích, động cơ học tập cho sinh viên; } \\
\text { hướng dẫn sinh viên phương pháp học tập bậc đại học }\end{array}$ & 4,10 & 0,71 \\
\hline $\begin{array}{l}\text { 33. Trường/Khoa có biện pháp quản lí hoạt động tự học và hoạt động học tập trên lớp của } \\
\text { sinh viên }\end{array}$ & 4,04 & 0,73 \\
\hline $\begin{array}{l}\text { 34. Sinh viên được tham gia lấy ý kiến phản hồi về hoạt động giảng dạy của giảng } \\
\text { viên các hoạt động hồ trợ đào tạo }\end{array}$ & 4,06 & 0,69 \\
\hline $\begin{array}{l}\text { 35. Các ý kiến phản hồi của sinh viên được sử dụng để điều chỉnh hoạt động giảng dạy của } \\
\text { giảng viên và các hoạt động đào tạo khác }\end{array}$ & 4,12 & 0,64 \\
\hline 36. Kết quả học tập của người học được thông báo kịp thời và quản lí theo quy định & 4,17 & 0,62 \\
\hline 37. Việc công nhận kết quả học tập của người học được thực hiện đúng quy chế & 4,09 & 0,72 \\
\hline 38. Văn bằng, chứng chỉ được cấp theo đúng quy định & 4,03 & 0,69 \\
\hline 39. Sinh viên tốt nghiệp đáp ứng mục tiêu đào tạo và yêu cầu của chuẩn đầu ra & 4,10 & 0,70 \\
\hline
\end{tabular}

Bảng 10. Thống kê kết quả khảo sát của cán bộ về QL đội ngũ cán bộ quản lí, nhân viên hỗ trợ hoạt động đào tạo

\begin{tabular}{lcc}
\hline Quản lý đội ngũ cán bộ QL, nhân viên hỗ trợ hoạt động đào tạo & ĐTB & $\begin{array}{c}\text { Độ lệ̣ch } \\
\text { chuẩn }\end{array}$ \\
\hline 40. Đội ngũ cán bộ QL có phẩm chất đạo đức, năng lực quản lí, lãnh đạo & 4,06 & 0,74 \\
41. Có đủ đội ngũ cố vấn học tập, nhân viên để thực hiện các hoạt động hổ trợ đào tạo & 4,05 & 0,69 \\
42. Chiến lược, chính sách đào tạo, bồi dưỡng đội ngũ cán bộ QL, giảng viên, nhân & 3,90 & 0,78 \\
viên rõ ràng và được triển khai thực hiện hiệu quả & 3,90 & 0,77 \\
43. Đội ngũ cán bộ, giảng viên và nhân viên được đảm bảo các quyền lợi theo quy định & 3,90 \\
\hline
\end{tabular}

Về QL môi trường học tập, co sở vật chất và trang thiết bị, thông số thống kê liên quan đến QL môi trường học tập, cơ sở vật chất và trang thiết bị cho thấy đánh giá của cán bộ chưa cao đối với hoạt động này. Trong các tiêu chí liên quan thì tiêu chí Thư viện có đầy đủ giáo trình, tài liệu phục vụ giảng dạy, học tập và NCKH có điểm trung bình cao nhất 3,85 với độ lệch chuẩn thấp nhất 0,85 . Được biết, trong thời gian qua, ĐHQGHN rất quan tâm đầu tư xây dựng đại học số hóa theo mô hình 2.0. Do đó, kết quả đánh giá này cũng khá phù hợp. Tiêu chí 46: Trang thiết bị dạy và học có đủ để hỗ trợ cho các hoạt động đào tạo có ĐTB $(3,70)$ thấp nhất trong nghiên cứu khảo sát này. Tuy nhiên, đây là kết quả đã thực hiện đầu năm 2016. Đến nay, ĐHQGHN đã triển khai đầu tư hoàn chỉnh hệ thống 160 phòng học thông minh. Tiêu chí 48: Kí túc xá, khu vui chơi, khu tập luyện thể thao đáp ứng nhu cầu của người học cũng là tiêu chí thấp thứ 2 từ dưới lên. 
Bảng 11. Thống kê kết quả khảo sát của cán bộ về QL môi trường học tập, cơ sở vật chất và trang thiết bị

\begin{tabular}{lcc}
\hline Quản lí môi trường học tập, cơ sở vật chất và trang thiết bị & ĐTB & $\begin{array}{c}\text { Độ lệ̉h } \\
\text { chuần }\end{array}$ \\
\hline 44. Thư viện có đầy đủ giáo trình, tài liệu phục vụ giảng dạy, học tập và NCKH & 3,85 & 0,85 \\
45. Phòng học, phòng thực hành, phòng thí nghiệm đáp ứng để phục vụ cho giảng & 3,78 & 0,94 \\
dạy, học tập và nghiên cứu & 3,70 & 0,91 \\
46. Trang thiết bị dạy và học có đủ để hỗ trợ cho các hoạt động đào tạo & 3,73 & 0,92 \\
47. Lớp học có đủ diện tích để tổ chức giảng dạy theo quy định & 3,71 & 0,98 \\
\hline 48. Kí túc xá, khu vui chơi, khu tập luyện thể thao đáp ứng nhu câuu của người học & \\
\hline
\end{tabular}

\section{2. Đánh giá của sinh viên đối với hoạt động quản lý đào tạo đại hoc của $Đ H Q G H N$}

Kết quả khảo sát tổng quát việc đánh giá của sinh viên về hoạt động QLĐT đại học tại ĐHQGHN được biểu diễn trên Hình 2 dưới đây.

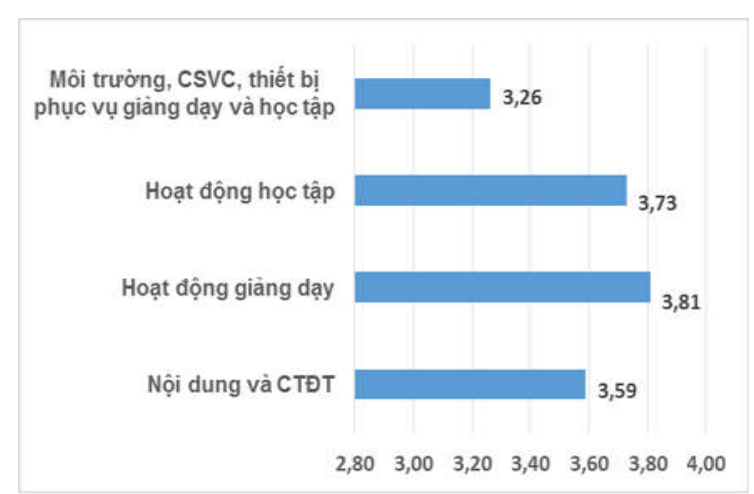

Hình 2. Đánh giá của sinh viên về hoạt động QLĐT đại học tại ĐHQGHN.

Kết quả phân tích số liệu cho thấy, nhân tố Hoạt động giảng dạy có ĐTB cao nhất $(3,81)$. Nhân tố Môi trường, cơ sở vật chất, thiết bị phục vụ giảng dạy và học tập có ĐTB thấp nhất $(3,26)$.

Kết quả phân tích tương quan của người học về hoạt động QLĐT của các đơn vị đào tạo trong ĐHQGHN có sự khác biệt với độ tin cậy $99 \%(\mathrm{p}<0,01)$. Điều đó có nghĩa là tuỳ vào đặc thù từng đơn vị đào tạo, với những nguồn lực, điều kiện khác nhau mà mỗi đơn vị có những thế mạnh riêng, từ đó đưa ra các giải pháp ưu tiên trong hoạt động QLĐT của mình, đồng thời cũng phản ánh tính thống nhất trong đa dạng của các đơn vị đào tạo ở ĐHQGHN.

Các kết quả này được trình bày và thảo luận chi tiết dưới đây, trong đó tập trung vào các điểm bất cập nhất, cần được quan tâm để cải thiện chất lượng đào tạo.
Về Nội dung và CT円T, hai tiêu chí được sinh viên đánh giá tốt nhất là Thông tin về CTĐT được cung cấp đầy đủ đến người học (ĐTB 3,74) và Nội dung CTĐT sát với mục tiêu đào tạo với ĐTB 3,68.

Về hoạt động giảng dạy, đánh giá của người học về hoạt động giảng dạy có 7 tiêu chí, trong tiêu chí có ĐTB cao nhất $(4,04)$ là về năng lực chuyên môn của đội ngũ giảng viên. Tiếp đến là tiêu chí về Hoạt động giảng dạy được triển khai đánh giá định kì theo quy định (ĐTB 3,97$)$. Điều này cũng rất phù hợp và cho thấy sự đánh giá chính xác của sinh viên đối với một cơ sở giáo dục đại học hàng đầu Việt Nam. Trong những năm qua, ĐHQGHN là một đại học tiên phong trong lĩnh vực đảm bảo và kiểm định chất lượng giáo dục.

Vì̀ hoạt động học tập, thông số thống kê liên quan đến hoạt động học tập qua đánh giá của người học ta thấy văn bằng, chứng chỉ là yếu tố đáp ứng nhu cầu việc làm của sinh viên và đó cũng là thành quả đánh giá quá trình trau dồi kiến thức của sinh viên nên yếu tố văn bằng, chứng chỉ được cấp theo quy định được các sinh viên quan tâm nhiều nhất (ĐTB 3,90). Ngoài ra thì người học và giảng viên phải kêt hợp với nhau để người học tiếp thu được kiến thức và người dạy truyền tải kiến thức thành công hơn nên ý kiến của sinh viên đối với công tác đào tạo cũng hết sức quan trọng, điều này thể hiện việc tôn trọng ý kiến sinh viên từ phía nhà trường và tiêu chí Sinh viên được tham gia lấy ý kiến phản hồi về hoạt động giảng dạy của $G V$ và các hoạt động hỗ trợ đào tạo có ĐTB cao nhất $(3,94)$ cho thấy đây là sự cần thiết trong hoạt động học tập theo đánh giá của sinh viên. 
Về môi truò̀ng, cơ sở vật chất, thiết bị phục vu giảng dạy và học tập qua đánh giá của người học cho thấy Môi trường, cơ sở vật chất, trang thiết bị của nhà trường luôn là mối quan tâm đối với sinh viên nhưng trong 5 tiêu chí được đánh giá thì người học lại đánh giá cao tiêu chí Thư viện (ĐTB 3,42). Chính vì vậy, đối với mỗi cơ sở giáo dục thì Thư viện cần có đầy đủ giáo trình, tài liệu phục vụ giảng dạy, học tập và nghiên cứu. Trong khi đó tiêu chí về Kí túc xá, khu vui chơi, khu tập luyện thể thao đáp ứng nhu cầu của người học lại có ĐTB thấp nhất $(3,11)$. Mặc dù hiện nay, ĐHQGHN đã đầu tư và có một khu thể thao đa năng bao gồm sân bóng và các phòng tập thể thao hiện đại tại trường Đại học Ngoại ngữ, nhưng có lẽ vẫn cần phải có nhiều khu như thế mới đáp ứng nhu cầu ngày càng lớn của người học.

\section{3. Ý kiến của người học về các vấn đề liên quan đến cá nhân}

Ngoài những nghiên cứu, khảo sát nhận định của cán bộ, sinh viên về hoạt động QLĐT ở ĐHQGHN, tác giả cũng quan tâm đến khảo sát một số chỉ số về lộ trình, định hướng học tập cũng như sự lựa chọn môn học của sinh viên. Những kết quả này có thể là nguồn tham khảo hữu ích trong hoạt động QLĐT đối với các đơn vị đào tạo.

Về lộ trình, định hướng học tập, qua kết quả khảo sát cho thấy trên $60 \%$ sinh viên được hỏi cho rằng họ có lộ trình, định hướng học tập rõ ràng như tự xác định được lộ trình, định hướng học tập của bản thân.
Về lựa chọn, đăng ki môn học,trên $70 \%$ người học cho rằng họ có tham gia hoặc tạo các nhóm bạn có cùng định hướng nghiên cứu/chuyên ngành, có lựa chọn và đăng kí môn học, các môn học có nội dung liên quan đến nhau trong cùng một kì.

\section{Nhận xét và thảo luận}

Dưới đây là một số so sánh kết quả nghiên cứu với kết quả kiểm định chất lượng giáo dục đại học.

Từ sơ đồ mô hình bảo đảm chất lượng cấp chương trình và cấp cơ sở giáo dục đại học của Mạng lưới các trường đại học ASEAN (AUN) cho thấy, các nhân tố khảo sát của nghiên cứu này hầu như rất gần với toàn bộ tiếp cận của kiểm định chương trình đào tạo (về chương trình đào tạo, hoạt động dạy và học, đánh giá sinh viên, chất lượng giảng viên và bộ phận phục vụ, chất lượng sinh viên và hỗ trợ sinh viên, điều kiện cơ sở vật chất, Hình 3); đồng thời, khảo sát này cũng đã ưu tiên tập trung vào mô-đun đảm bảo chất lượng về việc thực hiện chức năng đào tạo của cơ sở giáo dục đại học (các nhân tố 13-17, Hình 4). Trong cách xây dựng phương pháp nghiên cứu, các chỉ số cũng có khả năng bao phủ các yếu tố quản trị chiến lược và quản trị hệ thống. Do đó, trước hết, có thể xem nghiên cứu này có cùng cách tiếp cận khảo sát, đánh giá chung về điều kiện đảm bảo chất lượng đào tạo.

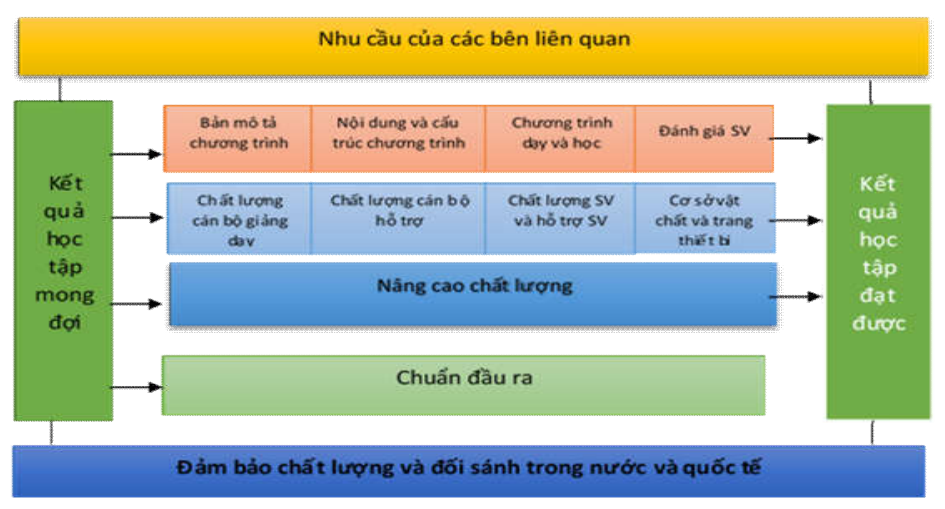

Hình 3. Mô hình kiểm định điều kiện đảm bảo chất lượng cấp chương trình đào tạo của AUN [1]. 


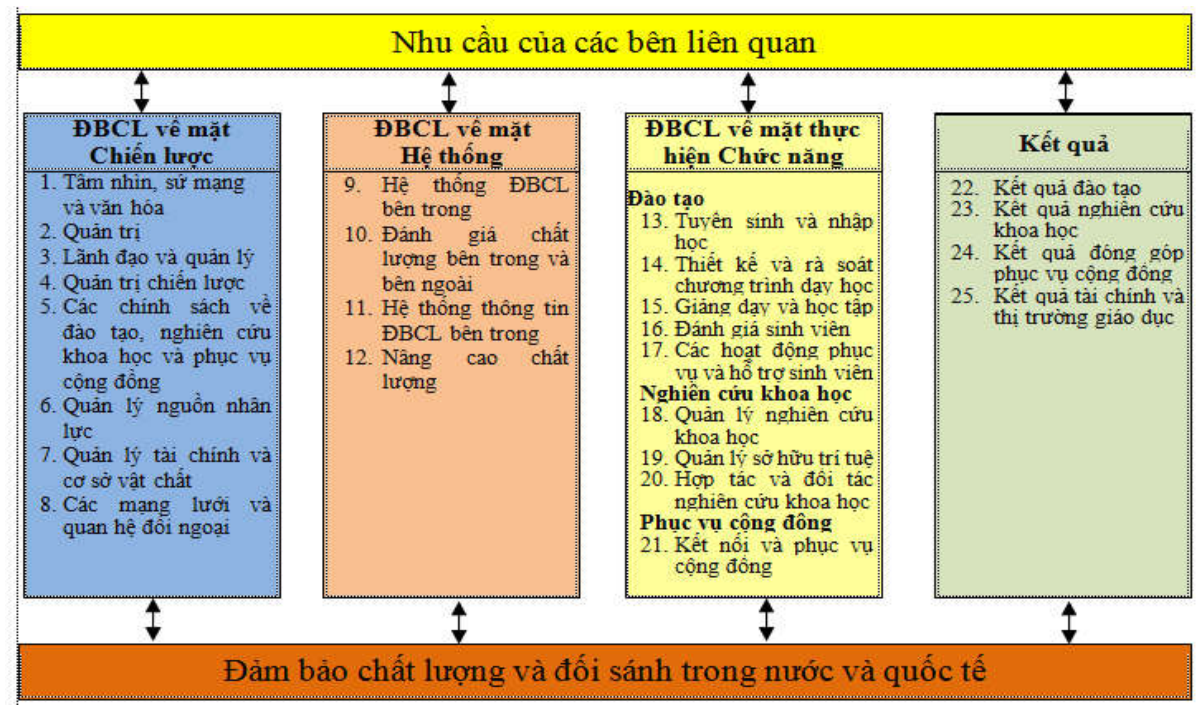

Hình 4. Mô hình kiểm định điều kiện đảm bảo chất lượng cấp cơ sở đào tạo của AUN [3].

Ngoài các đánh giá của các đoàn đánh giá ngoài của $A U N$ cho các chương trình đào tạo, gần đây (đầu năm 2017), AUN cũng vừa tổ chức đánh giá đảm bảo chất lượng cấp cơ sở giáo dục đại học cho Trường Đại học Khoa học Tự nhiên (ĐHKHTN), ĐHQGHN. Theo kết quả đánh giá này [8], bên cạnh các điểm mạnh (là chủ yếu), các chuyên gia đánh giá ngoài của AUN cũng đã khuyến nghị trường ĐHKHTN quan tâm cải thiện một số yếu tố, các khuyến nghị này rất phù hợp với kết quả khảo sát của nghiên cứu này.

Từ việc so sánh kết quả khảo sát với kết quả kiểm định chất lượng giáo dục của AUN, cho phép tác giả đưa ra một số khuyến nghị đối với ĐHQGHN trong quản lí hoạt động đào tạo.

Thư nhất, về mục tiêu và chiến lược phát triển. Bên cạnh viêc xây dựng mục tiêu, chiến lược phát triển có tính học thuật vững chắc, cần quan tâm điều chỉnh tầm nhìn phù hợp với các bên liên quan, tìm hiểu nhu cầu xã hội để đào tạo nhằm tăng cơ hội việc làm cho sinh viên sau tốt nghiệp, tăng cường phát triển hợp tác doanh nghiệp phù hợp với phát triển nền kinh tế thị trường định hướng xã hội chủ nghĩa. (Điều này khá phù hợp với kết quả khảo sát các tiêu chí về mục tiêu và chiến lược phát triển, trong đó tiêu chí 2 có ĐTB thấp).
Thú hai, về nội dung CTĐT. Cần rút ngắn chu kì rà soát CTÐТ; nội dung CTÐT gắn với với nhu cầu của thị trường lao động, đảm bảo cho sự phát triển cộng sinh. (Đây chính là điểm yếu của Tiêu chí 7 về yêu cầu thường xuyên cập nhật và điều chỉnh phù hợp nội dung CTĐT).

Thư $b a$, về công tác tuyển sinh. Cần thiết lập hệ thống thu thập và phân tích thông tin về thị trường và doanh nghiệp, phân tích tác động của các tiêu chí tuyển sinh, các kết quả học tập của sinh viên, nhất là tuyển sinh các ngành khoa học cơ bản, tăng cường tuyển sinh viên nước ngoài. Tiếp tục thực hiện lộ trình tuyển sinh theo phương thức đánh giá chung sang đánh giá năng lực chuyên biệt nhằm tuyển chọn sinh viên phù hợp năng lực và ngành nghề đào tạo, nâng cao chất lượng đào tạo và đáp ứng thị trường lao động. Khuyến nghị này chính là nội dung của Tiêu chí 11 về yêu cầu đối với kế hoạch tuyển sinh đã khảo sát.

Thư $t u$, về tổ chức thực hiện đào tạo. Kết quả nghiên cứu đối với Tiêu chí 15 về việc phân định chức năng, trách nhiệm và quyền hạn của các bộ phận, cán bộ quản lí, giảng viên và nhân viên cũng phù hợp với các khuyến nghị của $A U N$ về việc cần bồ sung một số chức năng cho các đơn vị, phòng, ban; tránh sự sự chồng chéo về chức năng hành chính giữa cấp ĐHQG và cấp trường thành viên; giảm bớt công việc 
và thủ tục hành chính cho giảng viên. Cần có quy định rõ ràng về phối hợp giữa các đơn vị trong nhà trường và khoa để thực hiện nhiệm vụ đào tạo một cách hiệu quả.

Thư năm, về hoạt động giảng dạy. Tăng cường việc nâng cao năng lực chuyên môn của giảng viên; thực hiện phân công giảng dạy theo chuyên môn được đào tạo; giảng viên tích cực đổi mới phương pháp giảng dạy theo đúng nội dung và thời lượng của CTĐT. Điều này phù hợp với khuyến nghị của $A U N$ về việc cần xác định một triết lí giáo dục, tăng cường bồi dưỡng nghiệp vụ sư phạm; nghiên cứu và chia sẻ các kinh nghiệm tốt. Đây chính là Tiêu chí 26 về đổi mới phương pháp giảng dạy. Lưu ý rằng, mặc dù chúng ta đang ở vào thời kì công nghiệp 4.0, công nghệ sẽ là yếu tố hỗ trợ rất lớn, theo khuyến cáo này, công nghệ giáo dục không có triết lí cũng sẽ là công nghệ không có khả năng kết nối và giao tiếp, không có "linh hồn".

Thú sáu, về hoạt động học tập của sinh viên. Sinh viên cần được hướng dẫn đầy đủ về CTĐT, các quy định về kiểm tra đánh giá và các quy định trong quy chế đào tạo, quy chế công tác HSSV. Theo AUN, cần rà soát đánh giá và củng cố thiết kế của hướng dẫn đánh giá thang bậc chất lượng học tập môn học (rubrics and barem) để đáp ứng các nguyên tắc kiểm tra đánh giá. Do vậy rất phù hợp với khuyến nghị và kết quả khảo sát theo tiêu chí 30 của nghiên cứu này.

Thư bảy, về phát triển đội ngũ cán bộ quản lí, giảng viên, nhân viên hỗ trợ. Cần làm tốt chiến lược, chính sách đào tạo, bồi dưỡng đội ngũ cán bộ QL, giảng viên, nhân viên cũng như đảm bảo các quyền lợi của họ (Tiêu chí 42). Theo AUN, cần rút ngắn thời hạn thăng thưởng cho cán bộ, giảng viên và nhân viên, cần quan tâm hơn tới việc đào tạo bồi dưỡng kĩ năng lãnh đạo, quản lí và tăng cường đầu tư nhiều hơn cho con người.

Cuối cùng, về môi trường học tập, cơ sở vật chất và trang thiết bị. Theo kết quả khảo sát của nghiên cứu này, thì tiêu chí 48 có ĐTB thấp nhất (kí túc xá, khu vui chơi, khu tập luyện thể thao đáp ứng nhu cầu của người học). Do vậy, cần đặc biệt quan tâm đến không gian học tập và các trang thiết bị học tập; chú trọng đầu tư các lớp học thông minh và hệ thống hỗ trợ học tập trực tuyến; cơ sở vật chất cần thân thiện hơn đối với sinh viên, quan tâm hơn đến đời sống sinh viên và không gian xã hội trong trường đại học theo định hướng xây dựng văn hóa chất lượng, trong đó thành tố môi trường tự nhiên (cảnh quan, cơ sở vật, thư viện, ký túc xá, an ninh trật tự, đời sống văn hóa-tinh thần) [9] cũng rất quan trọng góp phần đảm bảo và nâng cao chất lượng các hoạt động đào tạo.

\section{Kết luận}

Nghiên cứu này quan tâm khảo sát vấn đề QLĐT nhằm tăng cường công tác đảm bảo chất lượng giáo dục. Bằng việc tổng hợp các nghiên cứu lí luận và thực tiễn, công trình đã đề xuất được mô hình đánh giá bao gồm 8 thang đo để đo lường các khái niệm nghiên cứu. Các thang đo được đánh giá thông qua hệ số tin cậy Cronbach Alpha và phương pháp phân tích nhân tố EFA rất hữu hiệu. Các kết quả thống kê đã chỉ ra được điểm mạnh và điểm yếu cho các nhân tố khảo sát. Mặc dù có cách tiếp cận riêng, lấy mẫu riêng và phân tích độc lập, nhưng các kết quả nghiên cứu cho những nhận định khá phù hợp với kết quả đánh giá bằng phương pháp kiểm định điều kiện đảm bảo chất lượng giáo dục theo các mô hình của AUN. Theo tiếp cận đa chiều, bài báo này khảo sát, đánh giá công tác QLĐT đại học ở ĐHQGHN một cách toàn diện (đa tham số) và khách quan (đa phương pháp, đa nguồn).

Cùng với các phân tích và so sánh này, công trình nghiên cứu đã đưa ra 8 khuyến nghị cần thiết để tiếp tục cải thiện các điều kiện đảm bảo chất lượng của ĐHQGHN.

\section{Lời cảm ơn}

Nhóm tác giả xin trân trọng cám ơn sự tài trợ của $\mathrm{DHQGHN}$. Nghiên cứu này nằm trong 
khuôn khổ đề tài khoa học và công nghệ cấp ĐHQGHN, mã số QG.15.29:

Phân tích, phai khá dũ liệu dạy học và thông tin phản hồi của sinh viên nhằm nâng cao chẩt lượng và hiệu quả quản lí đào tạo.

Nhóm tác giả cũng xin chân thành cám ơn sự đóng góp ý kiến của các nhà quản lí, cán bộ, giảng viên và sinh viên ĐHQGHN trong quá trình thực hiện nghiên cứu.

\section{Tài liệu tham khảo}

[1] Guide to AUN-QA Assessment at Programme Level published by ASEAN University Network (AUN), version 3.0 (2015).

[2] The World Declaration on Higher Education for the Twenty First Century: Vision and Action, UNESCO (October 1998).
[3] Guide to AUN-QA Assessment at Institutional Level published by ASEAN University Network (AUN), version 2.0 (2016).

[4] Nguyễn Thị Mỹ Lộc (chủ biên, 2004), Một số vấn đề về giáo dục đại học. Nxb Đại học Quốc gia Hà Nội.

[5] Nguyễn Thanh Bình (2005), Lí luận Giáo dục học Việt Nam, Nxb Đại học Sư phạm.

[6] Ranking Web of Universites, http://www.webometrics.info/en

[7] World University Rankings \& Reviews, http://www.4icu.org/

[8] $1^{\text {st }}$ AUN-QA Institutional Assessment VNU University of Science Hanoi, Vietnam - Reports, (January 2017). Lưu hành nội bộ.

[9] Lê Đức Ngọc, Trịnh Thị Vũ Lê, Nguyễn Thị Ngọc Xuân (2012), Bàn về mô hình văn hóa chất lượng cơ sở giáo dục đại học, Tạp chí Quản lí Giáo dục số (34) 3-2012.

\title{
Multi-dimensional Approach to Academic Affairs Management at Vietnam National University, Hanoi
}

\author{
Vuong Thi Phuong Thao ${ }^{1}$, Phan Xuan Hieu ${ }^{2}$ \\ ${ }^{1} V N U$ Centre for Educational Testing \\ ${ }^{2}$ VNU University of Engineering and Technology
}

\begin{abstract}
This paper examines and evaluates the undergraduate academic affair management at Vietnam National University, Hanoi using a comprehensive (multi-parameter) and objective (multidimensional) method. Specifically, the staff's assessment was conducted through eight factors: 1) Objectives and training plans; 2) Training programs management; 3) Admissions; 4) Training organization; 5) Lecturers teaching management; 6) Students learning activities management; 7) Management board and assistance staff support for training activities; and 8) Learning environment, facilities and equipment management. Students' assessment was implemented through four factors: 1) Training programs content; 2) Teaching activities; 3) Training activities; and 4) The environment, facilities and equipment for teaching and learning. The results of the study have been strengthened by the comparison with the results of AUN-QA experts' quality assurance assessment. Eight recommendations have also been made to enhance training quality assurance.
\end{abstract}

Keywords: Multi-dimensional approach, academic affair management, education accreditation, Vietnam National University, Hanoi. 